\title{
Dark matter from one-flavor SU(2) gauge theory
}

\author{
Anthony Francis, Renwick James Hudspith, Randy Lewis*, Sean Tulin \\ Department of Physics and Astronomy, York University, Toronto, Ontario, M3J 1P3, Canada \\ E-mail: afrancis.heplat@googlemail.com, \\ renwick.james.hudspith@googlemail.com, randy. lewis@yorku.ca, \\ stulineyorku.ca
}

SU(2) gauge theory with a single fermion in the fundamental representation is a minimal nonAbelian candidate for the dark matter sector, which is presently missing from the standard model. Having only a single flavor provides a natural mechanism for stabilizing dark matter on cosmological timescales. Preliminary lattice results are presented and discussed in the context of dark matter phenomenology.

34th annual International Symposium on Lattice Field Theory

24-30 July 2016

University of Southampton, UK

${ }^{*}$ Speaker. 


\section{Motivation}

As the standard model of particle physics does not explain all of the mass in galaxies and galaxy clusters, the existence of dark matter is inferred [1,2]. The dark sector could be a nonAbelian gauge theory, and the lattice approach is a valuable tool for studying dark matter candidates of this type $[3,4,5,6,7,8,9,10,11,12,13,14]$. For a recent review, see [15].

In the present work, we consider a minimal non-Abelian gauge theory for dark matter, namely SU(2) with a single fermion in the fundamental representation. It has similarities to QCD but also interesting differences, as will be discussed below. Of particular relevance to dark matter phenomenology is the fact that this simple theory can stabilize its dark matter candidate in a natural way, in contrast to $\mathrm{SU}(2)$ with two or more fundamental fermions for example, as discussed in section 5 .

\section{Basic theory}

The Lagrangian for SU(2) gauge theory with one Dirac flavor can be written as

$$
\mathscr{L}=-\frac{1}{4} F_{\mu \nu}^{a} F^{a \mu \nu}+i \bar{Q} \gamma^{\mu} D_{\mu} Q+m Q^{T} C E Q
$$

where

$$
Q=\left(\begin{array}{c}
\chi_{L} \\
C \bar{\chi}_{R}^{T}
\end{array}\right), \quad E=\left(\begin{array}{cc}
0 & 1 \\
-1 & 0
\end{array}\right)
$$

and $C$ is the charge conjugation matrix. The dark fermion field $Q$ has no standard model quantum numbers, but in a later section we will describe its interaction with the standard model through couplings to the Higgs sector.

The Lagrangian in eq. (2.1) has an unbroken global SU(2), namely

$$
Q \rightarrow e^{i \sum_{i=1}^{3} T_{i} \alpha_{i}} Q
$$

which is a generalization of baryon number. In a theory with $N_{f}>1$ fermion flavors, the global $\mathrm{SU}(2)$ would be $\mathrm{SU}\left(2 N_{f}\right)$. For lectures describing multi-flavor SU(2) gauge theory, see [16].

For $m=0$, the Lagrangian in eq. (2.1) also has an unbroken (but anomalous) global U(1), namely

$$
Q \rightarrow e^{i \beta} Q
$$

like the axial U(1) in QCD. We expect this U(1) symmetry to be broken dynamically by a mass-like vacuum expectation value.

The particle spectrum of one-flavor SU(2) will include mesons, baryons and glueballs, all of which must appear as multiplets of the global SU(2). The simplest creation operators for mesons have the form $\bar{Q} \Gamma Q$. The simplest creation operators for baryons have the form $Q^{T} C \Gamma E Q$ where $C$ is the charge conjugation matrix and $E$ was defined in eq. (2.2). One important example is $\bar{Q} \gamma_{5} Q$ which is a singlet denoted by $\eta$. Another important example is $\bar{Q} \gamma_{\mu} Q$ which is one entry in a triplet where the other two entries are the baryon and anti-baryon; we will name this triplet $\rho^{ \pm, 0}$. 


\begin{tabular}{c|cc}
\hline$\beta$ & 2.2 & 2.309 \\
lattice dimensions & $20^{3} \times 56$ & $28^{3} \times 56$ \\
number of configurations & 2000 & 1540 \\
acceptance & $73 \%$ & $74 \%$ \\
unitary $a m_{\text {bare }}$ & -0.865 & -0.76 \\
partially quenched $a m_{\text {bare }}$ & $-0.845,-0.855$ & $-0.74,-0.75$ \\
average plaquette & 0.5989 & 0.6255 \\
$a w_{0}$ & $1.430(5)$ & $1.956(7)$ \\
$m_{V} L$ & $9.0(2)$ & $8.8(2)$ \\
\hline
\end{tabular}

Table 1: Lattice parameters for each of our two lattice ensembles.

\section{Preliminary lattice explorations}

Our lattice studies use a standard discretization of eq. (2.1): the plaquette gauge action and the Wilson fermion action. This is achieved efficiently by using an up-to-date version of the HiRep code [17]. The lattice action contains two parameters: the inverse gauge coupling $\beta$ and the bare fermion mass $m_{\text {bare }}$. We have generated two ensembles with the $\beta$ values tuned to give comparable physical volumes, and for each ensemble we calculated propagators at the sea fermion mass as well as propagators at additional valence fermion masses. These partially quenched results are used to study fermion mass dependences. Further details of our ensembles are displayed in Table 1.

The hadron mass spectrum at the smaller lattice spacing, neglecting disconnected diagrams, is shown in figure 1. This absence of disconnected diagrams removes the anomaly contribution to $m_{\eta}^{2}$, resulting in a linear dependence on the bare fermion mass as if it were a Goldstone boson. When disconnected diagrams are included, the $\eta$ should not be an authentic Goldstone boson due to the anomaly. Note that disconnected diagrams have recently been studied in the two-flavor theory [14].

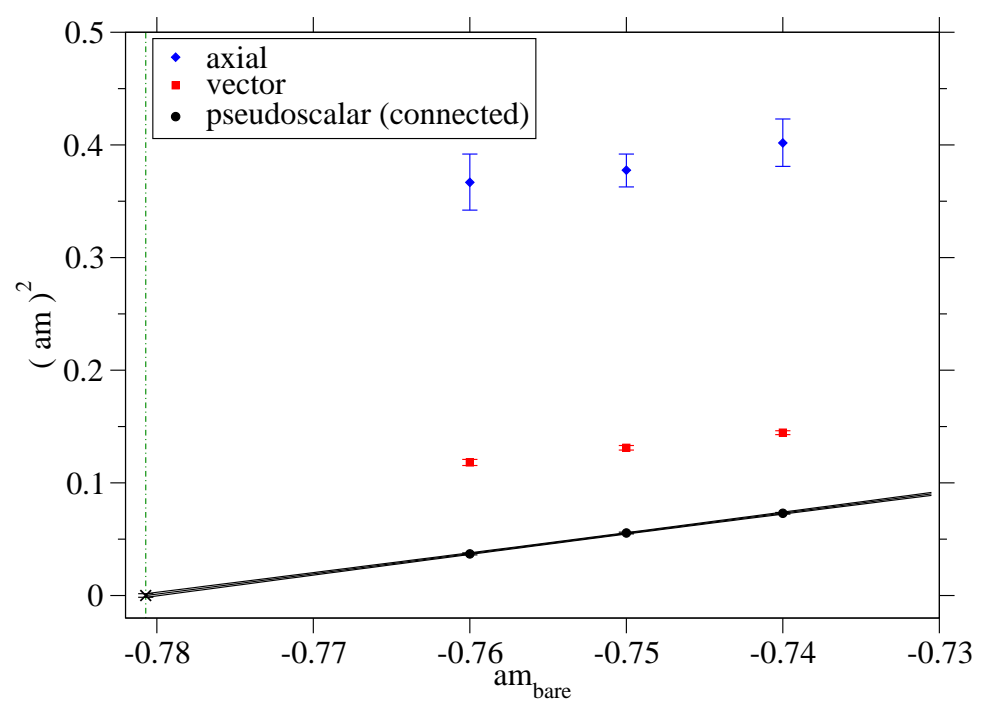

Figure 1: Three hadron masses (squared) as a function of the bare fermion mass at $\beta=2.309$. 
As in QCD, the axial meson is heavier than the vector which is heavier than the pseudoscalar. Recall that the lightest baryon and anti-baryon are exactly degenerate with the vector meson due to the global SU(2). Linear extrapolations of the vector and axial masses are displayed in figure 2. The vertical dashed line is where the connected part of $m_{\eta}=0$, and in that limit the axial meson mass is approximately twice as large as the vector meson mass.

Some of the raw correlation functions that generated our hadron mass results are presented in figure 3. We use Coulomb gauge fixed wall sources and perform contractions with a wall sink or a point sink. The resulting correlation functions have small statistical uncertainties and are dominated by the ground state, so one-state fits give precise hadron masses.

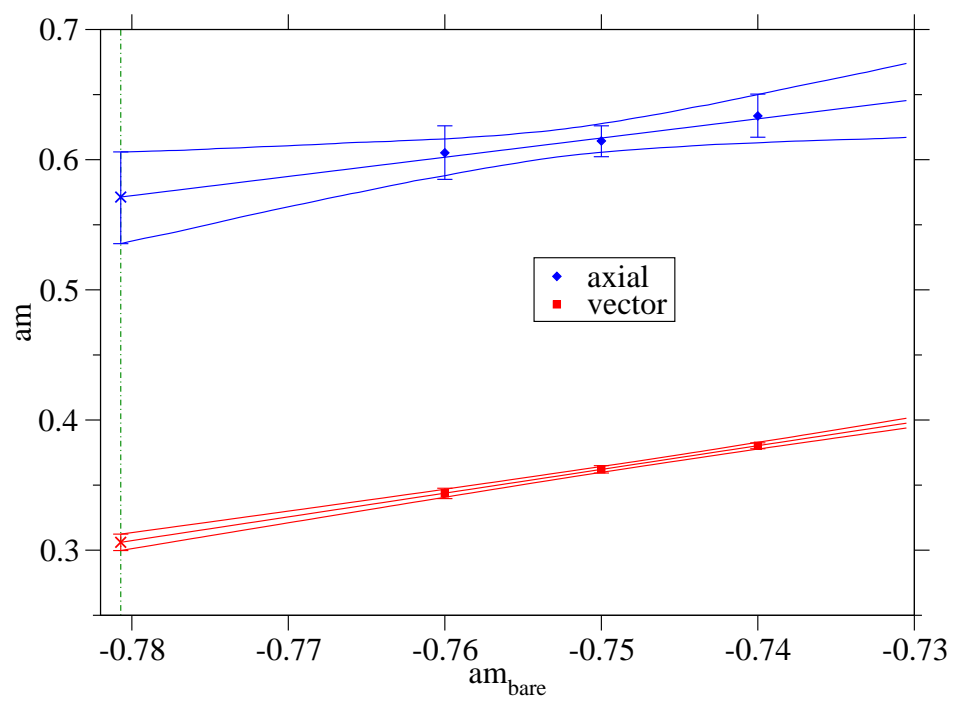

Figure 2: Linear extrapolations for the vector and axial vector meson masses as a function of the bare fermion mass at $\beta=2.309$.
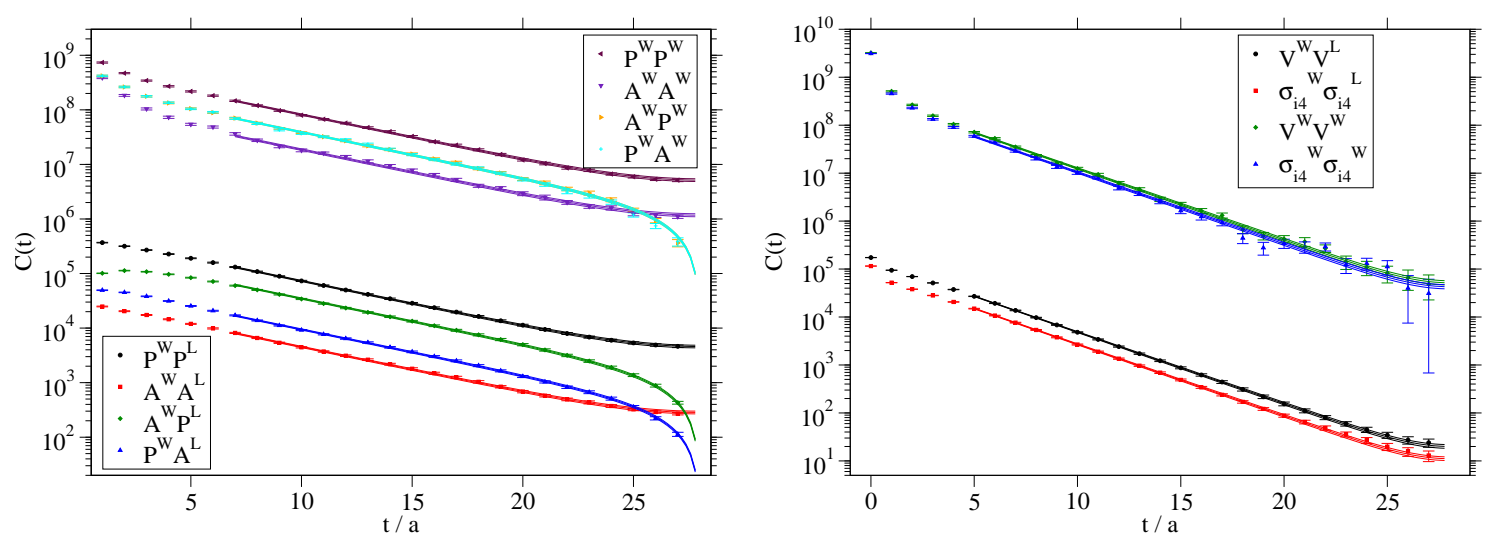

Figure 3: Correlation functions at $\beta=2.309$ and $a m_{\text {bare }}=-0.76$ for connected pseudoscalar (left panel) and vector (right panel). L denotes a local point sink and $\mathrm{W}$ is a Coulomb gauge fixed wall. $\mathrm{P}, \mathrm{A}, \mathrm{V}$ and $\sigma_{i 4}$ are conventional Dirac bilinears. 


\section{A large $N_{c}$ limit}

The standard limit of $\mathrm{SU}(2) \rightarrow \mathrm{SU}(3) \rightarrow \mathrm{SU}(4) \rightarrow \ldots$ is not useful in the present context because the global $\mathrm{SU}(2)$ symmetry that shapes the spectrum of $\mathrm{SU}(2)$ gauge theory does not exist for any $N_{c}>2$. However, $\mathrm{SU}(2)$ gauge theory is also $\mathrm{Sp}(2)$ gauge theory, and the global $\mathrm{SU}(2)$ symmetry is indeed present for any $\operatorname{Sp}\left(N_{c}\right)$ gauge theory with even $N_{c}$. Therefore the useful limit is $\mathrm{Sp}(2) \rightarrow \mathrm{Sp}(4) \rightarrow \mathrm{Sp}(6) \rightarrow \ldots$. The $\eta$ becomes massless as $N_{c} \rightarrow \infty$ and $m_{Q} \rightarrow 0$. In this double limit, the global $\mathrm{U}(1)$ is only broken dynamically and the $\eta$ is its Goldstone boson.

It might seem surprising that the global $\mathrm{SU}(2)$ can really remain unbroken as $N_{c} \rightarrow \infty$. A baryon with fermion content $X=\sum_{i, j, \cdots k=1}^{N_{c}} Q_{i} Q_{j} \ldots Q_{k}$ cannot be inside the same multiplet as a meson with fermion content $M=\sum_{i=1}^{N_{c}} \bar{Q}_{i} Q_{i}$ since they clearly contain differing numbers of fermions. The resolution is that $X$ is actually a collection of baryons; an individual baryon is a two-fermion object in $\operatorname{Sp}\left(N_{c}\right)$ gauge theory: $B=\sum_{i=1}^{N_{c}} \sum_{j=1}^{N_{c}} Q_{i} E_{i j} Q_{j}$. In this way, the global $\mathrm{SU}(2)$ remains unbroken. Baryons $B$ and mesons $M$ remain degenerate.

\section{Coupling to the standard model}

Dark matter appears to have no direct couplings to the standard model gauge theories, but there can be couplings to the standard model Higgs. In the one-flavor SU(2) theory, the leading interaction is a dimension 5 term:

$$
\delta \mathscr{L} \propto \frac{1}{\Lambda} \bar{Q} \gamma_{5} Q h^{\dagger} h
$$

which will couple to the dark $\eta$ meson. $\Lambda$ denotes the scale of new BSM physics. For the dark $\rho$ triplet, the leading Higgs interaction is dimension 6:

$$
\delta \mathscr{L} \propto \frac{1}{\Lambda^{2}} \bar{Q} \gamma_{\mu} Q h^{\dagger} \nabla^{\mu} h .
$$

Our dark matter candidate is the $\rho$, which can be stable for the life of the universe: recall for example that standard model proton decay is also a dimension 6 interaction. The dark $\eta$ will not be so long lived. It should be noted that $\rho$ stability is a consequence of having a single fermion flavor: with multiple flavors there are dimension 5 operators for $\rho$ decay.

Without assuming parity conservation in the dark sector, the mass terms for the dark fermion are

$$
\delta \mathscr{L}=-m_{4} \cos \theta_{4} \bar{Q} Q-m_{4} \sin \theta_{4} \bar{Q} i \gamma_{5} Q-\frac{v^{2}}{\Lambda} \cos \theta_{5} \bar{Q} Q\left(1+\frac{h}{v}\right)^{2}-\frac{v^{2}}{\Lambda} \sin \theta_{5} \bar{Q} i \gamma_{5} Q\left(1+\frac{h}{v}\right)^{2}
$$

where $v=246 \mathrm{GeV}$ is the electroweak scale. Notice that the dark fermion gets mass from 2 sources: from BSM at dimension 4 and through the Higgs at dimension 5.

The mass terms can be written more compactly as $\delta \mathscr{L}=m \bar{Q}_{\mathrm{tw}} Q_{\mathrm{tw}}$ where the twisted field is $Q_{\mathrm{tw}} \equiv e^{i \gamma_{5} \alpha / 2} Q$. Our use of the standard Wilson lattice action corresponds to implicitly untwisting the original mass terms in eq. (5.3). Vector and axial hadrons are invariant under $Q \rightarrow Q_{\mathrm{tw}}$.

The current experimental bound on invisible Higgs decays, $\Gamma(h \rightarrow Q \bar{Q})<1.2 \mathrm{MeV}$, provides a lower bound on the BSM scale in our dark sector, as shown in figure 4. The typical scale is seen to be tens of $\mathrm{TeV}$. 


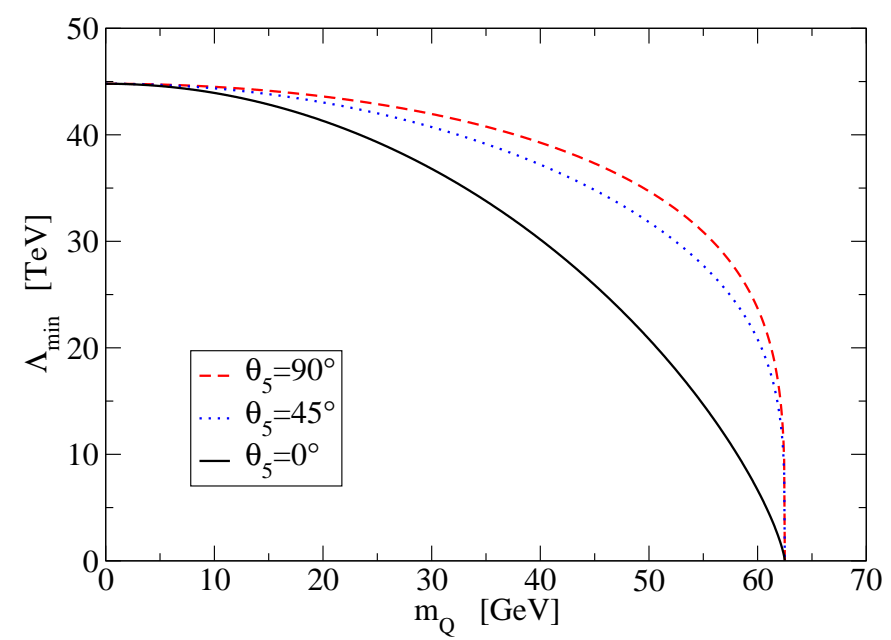

Figure 4: The minimum BSM scale as a function of dark fermion mass, for various choices of $\theta_{5}$ in eq. (5.3).

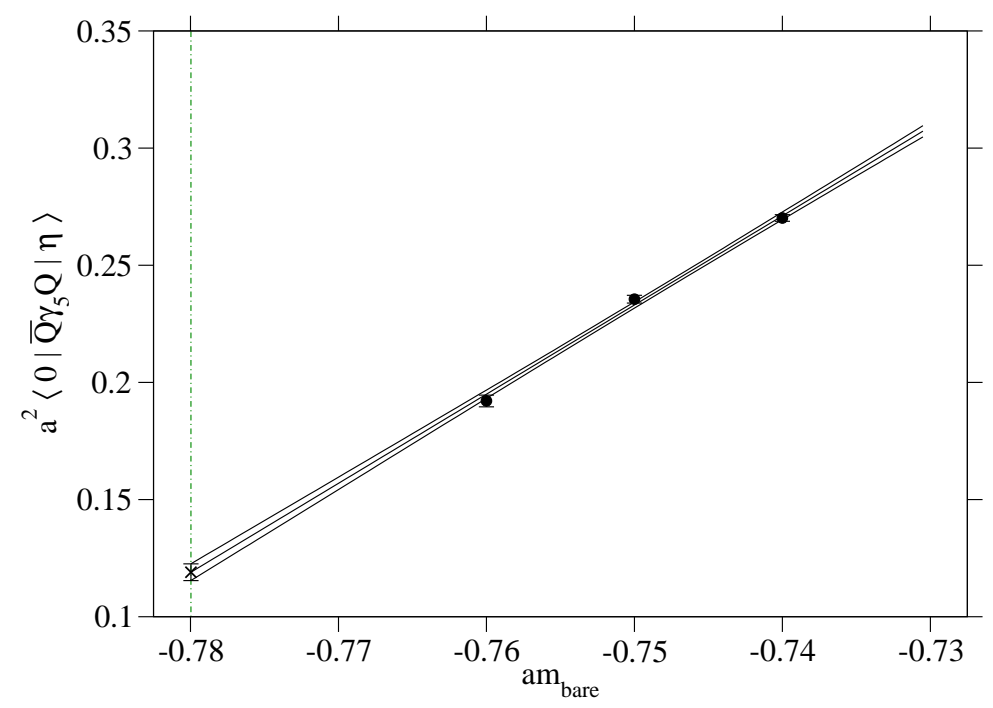

Figure 5: The connected part of the $\eta$ decay amplitude at $\beta=2.309$.

The only decays from the dark sector into standard model particles are through Higgs bosons. With the $\rho$ as our, essentially stable, dark matter candidate, an additional constraint comes from big bang nucleosynthesis, which requires the $\eta$ lifetime to be less than about 1 second [18]. This means that a lattice determination of $\left\langle 0\left|\bar{Q} \gamma_{5} Q\right| \eta\right\rangle$ would provide a bound on $\frac{\sin \theta_{5}}{\Lambda}$. A calculation for $m_{\eta} \ll m_{h}$ gives

$$
\Gamma_{\eta}=\left|\left\langle 0\left|\bar{Q} \gamma_{5} Q\right| \eta\right\rangle\right|^{2} \frac{m_{\eta} \sin ^{2} \theta_{5}}{2 \pi \Lambda^{2} m_{h}^{4}} \sum_{f \in S M} m_{f}^{2}\left(1-\frac{4 m_{f}^{2}}{m_{\eta}^{2}}\right)^{3 / 2}
$$

A complete lattice calculation must include the disconnected diagrams, but a proof of concept is given in figure 5 , which shows the matrix element obtained from a lattice calculation of the connected part only. 


\section{Outlook}

One-flavor SU(2) gauge theory provides a minimal non-Abelian dark sector. It has a global $\mathrm{SU}(2)$ to stabilize dark matter (here called the $\rho$ ), and no dark matter decay at dimension 5. One Goldstone boson should emerge for $\operatorname{Sp}\left(N_{c} \rightarrow \infty\right)$, and disconnected diagrams must be computed to arrive at a complete lattice calculation.

One phenomenologically important topic left for future work is direct detection, which entails $\rho$ scattering from a standard model nucleon. Another is the relic density, which requires a lattice calculation that can determine whether the $\rho$ is heavier or lighter than the $\eta$. In particular, $m_{\rho}>m_{\eta}$ could have a relic density governed by $\rho \rho \rightarrow \eta \eta$ or asymmetric dark matter, whereas $m_{\rho}<m_{\eta}$ could be governed by $\rho \rho \rho \rightarrow \rho \rho$.

\section{Acknowledgments}

We are grateful to Claudio Pica for help in the initial stages of this work, and for providing access to the HiRep code. Calculations were performed on Compute Canada's GPC machine at SciNet. This work was supported in part by NSERC of Canada.

\section{References}

[1] J. C. Kapteyn, Astrophys. J. 55, 302 (1922).

[2] F. Zwicky, Helv. Phys. Acta 6, 110 (1933).

[3] R. Lewis, C. Pica and F. Sannino, Phys. Rev. D 85, 014504 (2012) [arXiv:1109.3513].

[4] A. Hietanen, C. Pica, F. Sannino and U. I. Søndergaard, Phys. Rev. D 87, 034508 (2013) [arXiv:1211.5021].

[5] T. Appelquist et al. [LSD Collaboration], Phys. Rev. D 88, 014502 (2013) [arXiv:1301.1693].

[6] A. Hietanen, R. Lewis, C. Pica and F. Sannino, JHEP 1412, 130 (2014) [arXiv:1308.4130].

[7] T. Appelquist et al. [LSD Collaboration], Phys. Rev. D 89, 094508 (2014) [arXiv:1402.6656].

[8] A. Hietanen, R. Lewis, C. Pica and F. Sannino, JHEP 1407, 116 (2014) [arXiv:1404.2794].

[9] W. Detmold, M. McCullough and A. Pochinsky, Phys. Rev. D 90, 115013 (2014) [arXiv:1406.2276].

[10] W. Detmold, M. McCullough and A. Pochinsky, Phys. Rev. D 90, 114506 (2014) [arXiv:1406.4116]].

[11] T. DeGrand, Y. Liu, E. T. Neil, Y. Shamir and B. Svetitsky, Phys. Rev. D 91, 114502 (2015) [arXiv:1501.05665]].

[12] T. Appelquist et al., Phys. Rev. Lett. 115, 171803 (2015) [arXiv:1503.04205].

[13] V. Drach, A. Hietanen, C. Pica, J. Rantaharju and F. Sannino, PoS LATTICE 2015, 234 (2016) [arXiv:1511.04370]].

[14] R. Arthur, V. Drach, A. Hietanen, C. Pica and F. Sannino, arXiv:1607.06654].

[15] G. D. Kribs and E. T. Neil, Int. J. Mod. Phys. A 31, 1643004 (2016) [arXiv:1604.04627].

[16] L. von Smekal, Nucl. Phys. Proc. Suppl. 228, 179 (2012) [arXiv:1205.4205].

[17] L. Del Debbio, A. Patella and C. Pica, Phys. Rev. D 81, 094503 (2010) [arXiv:0805.2058]].

[18] G. Gamow, Phys. Rev. 70, 572 (1946); Phys. Rev. 74, 505 (1948). 